\title{
Impact of Sarcopenia on Erectile Function after Nerve-Sparing Robot-Assisted Radical Prostatectomy
}

\author{
Yosuke Mitsui $^{1}$ (D) Takuya Sadahira $^{1}$ (D), Yuki Maruyama ${ }^{(\mathbb{D})}$, Ryota Sato ${ }^{2}$ (D) Acosta Gonzalez Herik Rodrigo $^{1}$ (D),

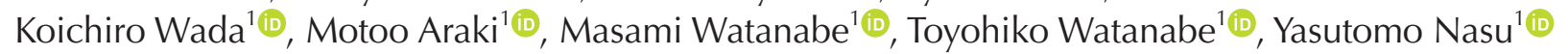 \\ ${ }^{1}$ Department of Urology, Okayama University Graduate School of Medicine, Dentistry and Pharmaceutical Sciences, Okayama, Japan, \\ ${ }^{2}$ Department of Internal Medicine, John A. Burns School of Medicine, University of Hawai'i at Manoa, Honolulu, HI, USA
}

Purpose: To determine the impact of sarcopenia on erectile functional outcomes after a nerve-sparing (NS) robot-assisted radical prostatectomy (RARP) using patient-reported validated questionnaires.

Materials and Methods: In this retrospective study, RARP was performed on 841 patients at Okayama University Hospital, of which 132 underwent NS RARP. Erectile functional outcomes were assessed using the 5-item version of the International Index of Erectile Function (IIEF-5) and the Expanded Prostate Cancer Index Composite before and 1, 3, 6, and 12 months after surgery. Automated measurement of skeletal muscle at L3 was achieved using volume analyzer software and normalizing for height $\left(\mathrm{cm}^{2} / \mathrm{m}^{2}\right)$ to calculate skeletal muscle index (SMI). Patients who had an IIEF-5 $\leq 4$ comprised the group with erectile dysfunction (ED), and those with an IIEF-5 $\leq 5$ made up the non-ED group.

Results: This study enrolled 95 patients of median age 65 years with a preoperative IIEF-5 of 16 . There were no significant differences between patients with and without sarcopenia among those with preoperative IIEF-5. Postoperatively, in the ED group, SMI and preoperative IIEF-5 were significantly lower than in the non-ED group. Multiple linear regression analysis revealed that (1) both SMI and preoperative IIEF-5 were independent predictors of ED, and (2) sarcopenia and preoperative IIEF-5 were predictors of ED at 12 months after NS RARP.

Conclusions: Patients with sarcopenia can have worse erectile functional outcomes after NS RARP. Sarcopenia and a lower preoperative IIEF-5 score may be predictive of postoperative ED.

Keywords: Erectile dysfunction; Prostatectomy; Prostatic neoplasms; Quality of life; Sarcopenia

This is an Open Access article distributed under the terms of the Creative Commons Attribution Non-Commercial License (http://creativecommons.org/licenses/by-nc/4.0) which permits unrestricted non-commercial use, distribution, and reproduction in any medium, provided the original work is properly cited.

\section{INTRODUCTION}

Sarcopenia, or the abnormal loss of skeletal muscle (SM) mass and strength, is one of the most important causes of functional decline and involves hormonal, nu- tritional, immunological, and physical activity changes [1]. Sarcopenia is associated with poor postoperative outcomes including surgical complications, increased infection risk, a long hospital length of stays, and can contribute to overall mortality during several types of

Received: Mar 10, 2020 Revised: Jul 14, 2020 Accepted: Jul 31, 2020 Published online Nov 16, 2020

Correspondence to: Takuya Sadahira (iD https://orcid.org/0000-0001-5685-3000

Department of Urology, Okayama University Graduate School of Medicine, Dentistry and Pharmaceutical Sciences, 2-5-1, Shikata-cho, Kita-ku, Okayama, 700-8558, Japan.

Tel: +81-86-231-7287, Fax: +81-86-231-3986, E-mail: t.sadahira@gmail.com 
surgery [1-4]. Currently, the association between sarcopenia and surgical outcomes after radical prostatectomy (RP) is poorly understood.

Sexual dysfunction, especially erectile dysfunction (ED), is a common and established complication of RP [5]. Although the oncologic outcome is the most important goal of RP, sexual dysfunction after RP typically has a negative effect on health-related quality of life (HRQoL). Urologists aim to choose and offer the best treatment option to their patients whilst considering both cancer control and quality of life (QoL) after treatment.

Risk factors for sexual dysfunction after RP in patients with prostate cancer $(\mathrm{PCa})$ have been reported for several studies [6-8]. Among them, patient age, preoperative erectile function, and nerve-sparing (NS) procedures are common factors influencing sexual function (SF) after prostatectomy [6-8]. However, sarcopenia has not been shown to be a risk factor for ED after RP.

Aging is one important factor that can lead to sarcopenia [9]. An ongoing increase in the number of elderly patients diagnosed with PCa poses a serious and emerging issue in cancer care with respect to QoL after RP. It can follow that better overall health care is vitally important to extending the survival of these types of patients. Sarcopenia has recently been investigated for its prognostic utility of treatment-related complications in cancer patients [1-4]. However, the impact of sarcopenia on SF after prostatectomy is unclear. We hypothesized that sarcopenia adversely affects erectile function recovery after RP. The aim of this study was to elucidate the effect of preoperative sarcopenia on sexual dysfunction after robot-assisted RP (RARP).

\section{MATERIALS AND METHODS}

\section{Patients}

We retrospectively identified 841 patients with $\mathrm{PCa}$ who had undergone RARP between October 2010 and May 2019 at Okayama University Hospital. Study inclusion criteria and patient selection are depicted in a flow diagram (Fig. 1). To remove the influence of surgical technique and experience, we selected only those cases performed by skillful surgeon with relatively same experience and finally 12 surgeons were included.

\section{Methods}

Computed tomography (CT) was performed using a

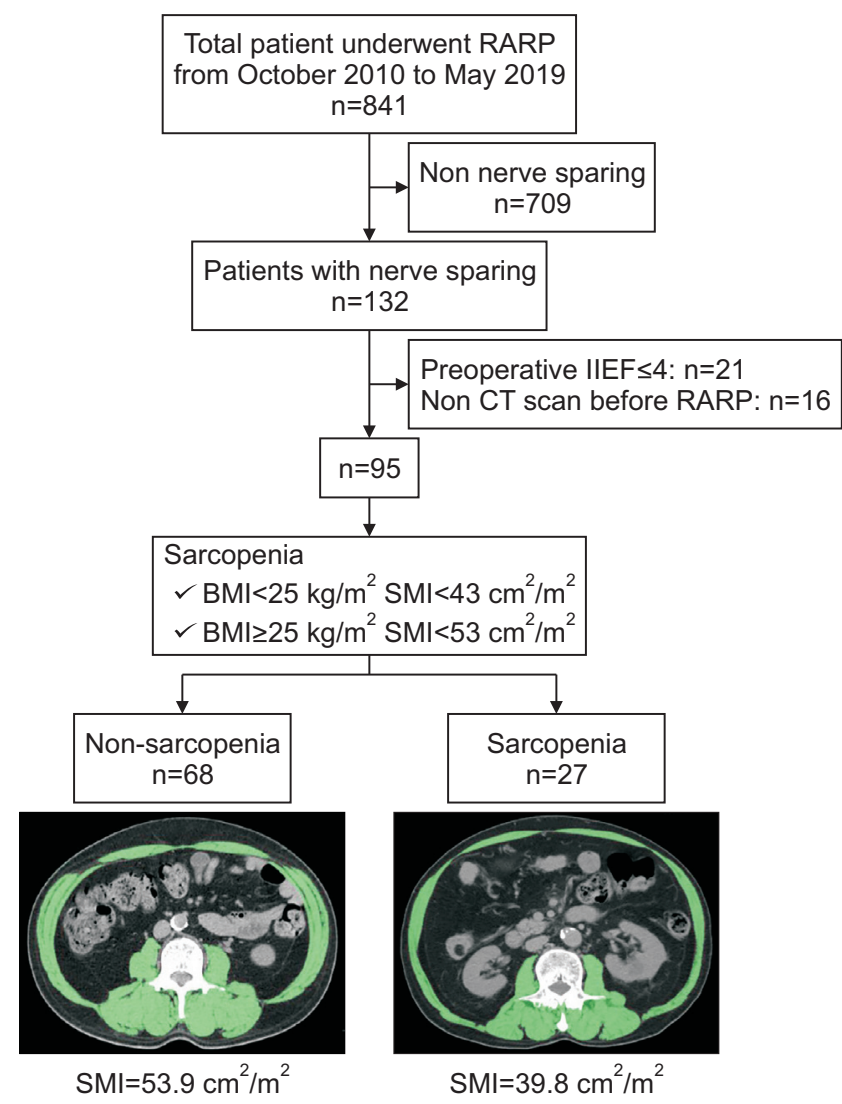

Fig. 1. Inclusion and exclusion criteria for this study. RARP: robotassisted radical prostatectomy, IIEF: International Index of Erectile Function, CT: computed tomography, BMI: body mass index, SMI: skeletal muscle index.

64-slice multi-detector scanner (Toshiba Medical Systems Corporation, Tochigi, Japan). Cross-sectional SM areas at the level of the third lumbar vertebra (L3) were measured automatically using the volume analyzer software Synapse Vincent version 4 (Fujifilm, Tokyo, Japan) [10] and threshold values of -29 to 150 Hounsfield units (HU) to distinguish muscle from other tissues. The SM area was normalized to height $\left(\mathrm{cm}^{2} / \mathrm{m}^{2}\right)$ to calculate the SM index (SMI). Simultaneously, visceral adipose tissue (VAT) and subcutaneous abdominal fat tissue areas at L3 were measured automatically using threshold values of -200 to -50 HU. Sarcopenia was defined, as described by Martin et al [11], as an SMI $<43 \mathrm{~cm}^{2} / \mathrm{m}^{2}$ for those with a body mass index (BMI) $<25$ $\mathrm{kg} / \mathrm{m}^{2}$ or a $\mathrm{SMI}<53 \mathrm{~cm}^{2} / \mathrm{m}^{2}$ for males with a $\mathrm{BMI} \geq 25$ $\mathrm{kg} / \mathrm{m}^{2}$, and as an $\mathrm{SMI}<41 \mathrm{~cm}^{2} / \mathrm{m}^{2}$ for female patients. To evaluate HR QoL, the comprehensive 50-item Expanded Prostate Cancer Index Composite (EPIC) instrument was used [12,13]. This questionnaire encompasses four different domains (urinary, bowel, sexual, and hormonal functions) and is designed to evaluate a patient's 
functioning on a normalized scale ranging from 0 to 100, with higher scores representing better QoL. The sexual domain of the EPIC consists of two subscales comprising SF and sexual bother [14]. EPIC was used to assess function before and after treatment received for PCa. Longitudinal HRQoL surveys were conducted at baseline (within 3 months before RARP) and at 2 weeks and 1, 3, 6, and 12 months after RARP using EPIC. Erectile function was also assessed according to the 5-item version of the International Index of Erectile Function (IIEF-5) score. Patients who had a low IIEF$5(\leq 4)$ were classified in the group with ED and those who had a relatively higher $\Pi \mathrm{EF}(\geq 5)$ were categorized in the group with non-ED. We chose a cutoff value of 5 because patients with a score less than 5 would be classified as having complete ED. In this study, we focused on sexual domain scores and their subscales after RARP.

\section{Surgical approach}

The surgeon first performed a pubic bone anchoring suture, followed by single-layer posterior reconstruction at the time of vesicourethral anastomosis. NS was undertaken in patients with PCa by intrafascial dissection using the veil technique [15]. Anterior or posterior approaches were used as follows. In the anterior approach, dissection of Retzius' space was started. The endopelvic fascia was incised laterally and carried distally to the apex of the prostate. The puboprostatic ligaments were incised. The bladder neck was incised between the bladder and prostate and the seminal vesicle and vas deferens were identified. In the posterior approach, the peritoneum in the pouch of Douglas was incised horizontally $2 \mathrm{~cm}$ above the rectum. The seminal vesicles and vas deferens were identified and the vas deferens were skeletonized, cauterized, and divided. The seminal vesicles were dissected free. Denonvilliers' fascia was incised in the midline and the rectum was dropped posteriorly off the prostate. Then, Retzius' space was dissected [16].

\section{Statistical analyses}

Patient characteristics and EPIC scores were statistically compared using the Wilcoxon test and Fisher's exact test, as deemed appropriate. The risk factors for $\mathrm{ED}$ at 12 months were analyzed using univariate and multivariate logistic regression analyses. Multivariate analysis included the variables determined to be sig- nificant in the univariate analysis. Statistical significance was set at $\mathrm{p}<0.05$ for all analyses. All statistical analyses were performed using JMP 13.2 software (SAS Institute, Cary, NC, USA).

\section{Ethics statement}

All procedures performed in this study involving human participants were in accordance with the ethical standards of the institutional research committee and with the 1964 Helsinki declaration and its later amendments (2013) or comparable ethical standards. This clinical study was approved by the Okayama University Institutional Review Board prior to study initiation (IRB registration no. 1004 and no. 2004-037).

\section{RESULTS}

\section{Patient characteristics}

A total of 95 patients were included in this retrospective study. Baseline patient characteristics, surgical procedures, and outcomes are reported (Table 1). Overall, 27/95 patients (28.4\%) were classified as sarcopenic. Although patients with sarcopenia have more hypertension (HT) and longer hospitalization stays than those without sarcopenia, there were no significant differences between patients with and without sarcopenia. A total of 14 patients were diagnosed with biochemical recurrence during follow-up. In 12 months, 7 patients were diagnosed with recurrence, 2 patients were treated with radiation therapy, 4 patients were treated with androgen blockage, and a patient chose active surveillance. Total 27 patients used phosphodiesterase-5 (PDE5) inhibitor after RARP (Supplement Table). All patients used PDE5 inhibitor on-demand.

\section{Change in health-related quality of life after robot-assisted radical prostatectomy}

Longitudinal changes in the EPIC sexual domain scores in the patients with and without sarcopenic are reported (Fig. 2). After RARP, the patients without sarcopenia had a better SF at 3 and 12 months compared with the patients who were sarcopenic. There were no significant differences in sexual bother scores between the two groups at any time point.

\section{Change in 5-item version of the International Index of Erectile Function after treatment}

The change in IIEF-5 from before RARP until 12 
Table 1. Patient characteristics

\begin{tabular}{|c|c|c|c|c|}
\hline Characteristic & Total cases $(n=95)$ & Non-sarcopenic $(n=68)$ & Sarcopenic $(n=27)$ & $\mathrm{p}$-value \\
\hline Age $(y)$ & $65(59-68)$ & $64.5(58-68)$ & $65(60-69)$ & 0.334 \\
\hline BMI $\left(\mathrm{kg} / \mathrm{m}^{2}\right)$ & $24.2(22.6-25.9)$ & $23.9(22.9-25.7)$ & $25.3(21.9-26.2)$ & 0.316 \\
\hline HT & $28(29.5)$ & $16(23.5)$ & $12(44.4)$ & 0.051 \\
\hline DM & $9(9.5)$ & $8(11.8)$ & $1(3.7)$ & 0.438 \\
\hline Prostate volume (mL) & $24.7(19.0-35.0)$ & $25(19.0-35.5)$ & $24(18.0-33.0)$ & 0.812 \\
\hline Initial PSA (ng/mL) & $7.5(5.1-9.3)$ & $7.2(5.0-9.5)$ & $8.0(5.9-9.3)$ & 0.287 \\
\hline Clinical T stage & & & & 0.177 \\
\hline$<\mathrm{cT} 1 \mathrm{c}$ & $32(33.7)$ & $22(32.4)$ & $10(37.0)$ & \\
\hline cT2a & $45(47.4)$ & $33(48.5)$ & $12(44.4)$ & \\
\hline cT2b & $5(5.3)$ & $5(7.4)$ & $0(0.0)$ & \\
\hline cT2c & $11(11.6)$ & $7(10.3)$ & $4(14.8)$ & \\
\hline cT3 over & $2(2.1)$ & $1(1.5)$ & $1(3.7)$ & \\
\hline ISUP grade group in prostate biopsy & & & & 0.254 \\
\hline Grade group 1 & $31(32.6)$ & $23(33.8)$ & $8(29.6)$ & \\
\hline Grade group 2 & $22(23.2)$ & $16(23.5)$ & $6(22.2)$ & \\
\hline Grade group 3 & $24(25.3)$ & $16(23.5)$ & $8(29.6)$ & \\
\hline Grade group 4 & $15(15.8)$ & $11(16.2)$ & $4(14.8)$ & \\
\hline Grade group 5 & $3(3.2)$ & $2(2.9)$ & $1(3.7)$ & \\
\hline \multicolumn{5}{|l|}{ CT measurement value } \\
\hline $\operatorname{VAT}\left(\mathrm{cm}^{2}\right)$ & $131(80-179)$ & $119(79-172)$ & 165 (98-194) & 0.067 \\
\hline SAT $\left(\mathrm{cm}^{2}\right)$ & $92(75-127)$ & $91.7(74-125)$ & $97(80-143)$ & 0.345 \\
\hline VAT/SAT ratio & $1.2(1.0-1.7)$ & $1.12(0.9-1.6)$ & $1.32(1.0-1.8)$ & 0.224 \\
\hline $\mathrm{SM}\left(\mathrm{cm}^{2}\right)$ & $139(129-152)$ & $143(132-156)$ & $128(120-138)$ & $<0.0001$ \\
\hline $\mathrm{SMI}\left(\mathrm{cm}^{2} / \mathrm{m}^{2}\right)$ & $49.7(46.0-55.5)$ & $53(47.9-56.3)$ & $45.8(42.1-49.2)$ & $<0.0001$ \\
\hline \multicolumn{5}{|l|}{ Operation data } \\
\hline Operation time (min) & $211(182-250)$ & $208(180-253)$ & 219 (189-234) & 0.426 \\
\hline $\mathrm{EBL}(\mathrm{mL})$ & $100(100-188)$ & $125(88-200)$ & $100(100-100)$ & 0.475 \\
\hline Anterior approach & $9(9.5)$ & $6(8.8)$ & $3(11)$ & 0.710 \\
\hline Nerve-sparing procedure & & & & $>0.999$ \\
\hline Unilateral & $76(80.0)$ & $54(79.4)$ & $22(81.5)$ & \\
\hline Bilateral & $19(20.0)$ & $14(20.6)$ & $5(18.5)$ & \\
\hline Inpatient days & $11(10-13)$ & $11(10-13)$ & $12(10-13)$ & 0.067 \\
\hline Preoperative IIEF-5 & $16(12-20)$ & $16(12-20)$ & $16(12-21)$ & 0.527 \\
\hline Lymph node dissection & & & & 0.709 \\
\hline Unilateral & $25(26.3)$ & $19(27.9)$ & $6(22.2)$ & \\
\hline Bilateral & $20(21.0)$ & $17(25)$ & $3(11.1)$ & \\
\hline Pathological T stage & & & & 0.265 \\
\hline $\mathrm{T} 2 \mathrm{a}$ & $13(13.7)$ & $10(14.7)$ & $3(11.1)$ & \\
\hline $\mathrm{T} 2 \mathrm{~b}$ & $6(6.3)$ & $4(5.9)$ & $2(7.4)$ & \\
\hline $\mathrm{T} 2 \mathrm{c}$ & $62(65.3)$ & $44(64.7)$ & $18(66.7)$ & \\
\hline$>\mathrm{T} 3 \mathrm{a}$ & $14(14.7)$ & $10(14.7)$ & $4(14.8)$ & \\
\hline
\end{tabular}

months after is shown in a waterfall plot (Fig. 3). This figure shows that patients with sarcopenia had a worse recovery in terms of erectile function.

\section{Association between sarcopenia and} erectile dysfunction 12 months after robotassisted radical prostatectomy

Table 2 shows the associations between sarcopenia and ED after surgery. Univariate analysis identified additional treatment, SMI and preoperative IIEF- 
Table 1. Continued

\begin{tabular}{lccc}
\hline \multicolumn{1}{c}{ Characteristic } & Total cases $(\mathbf{n = 9 5 )}$ & Non-sarcopenic $(\mathbf{n}=68)$ & Sarcopenic $(\mathbf{n}=\mathbf{2 7})$ \\
\hline ISUP grade group & & & $2(7.4)$ \\
Grade group 1 & $15(15.8)$ & $13(19.1)$ & $15(55.6)$ \\
Grade group 2 & $41(43.2)$ & $26(38.2)$ & $4(14.8)$ \\
Grade group 3 & $21(22.1)$ & $17(25)$ & $2(7.4)$ \\
Grade group 4 & $6(6.3)$ & $4(5.9)$ & $4(14.8)$ \\
Grade group 5 & $12(12.6)$ & $8(11.8)$ & $1(3.7)$ \\
Recurrence during post 12 months & $7(7.4)$ & $6(8.8)$ & 2.2 \\
Time for recurrence months & $8.1(5.4-9.1)$ & $8.3(6.9-9.4)$ & $1(3.7)$ \\
Additional treatment & $6(6.3)$ & $5(7.4)$ & 0.669 \\
\hline
\end{tabular}

Values are presented as median (interquartile range) or number (\%).

BMI: body mass index, HT: hypertension, DM: diabetes mellitus, PSA: prostate-specific antigen, ISUP: International Society of Urological Pathology, CT: computed tomography, VAT: visceral adipose tissue, SAT: subcutaneous adipose tissue, SM: skeletal muscle, SMI: skeletal muscle index, EBL: estimated blood loss, IIEF-5: 5-item version of the International Index of Erectile Function.
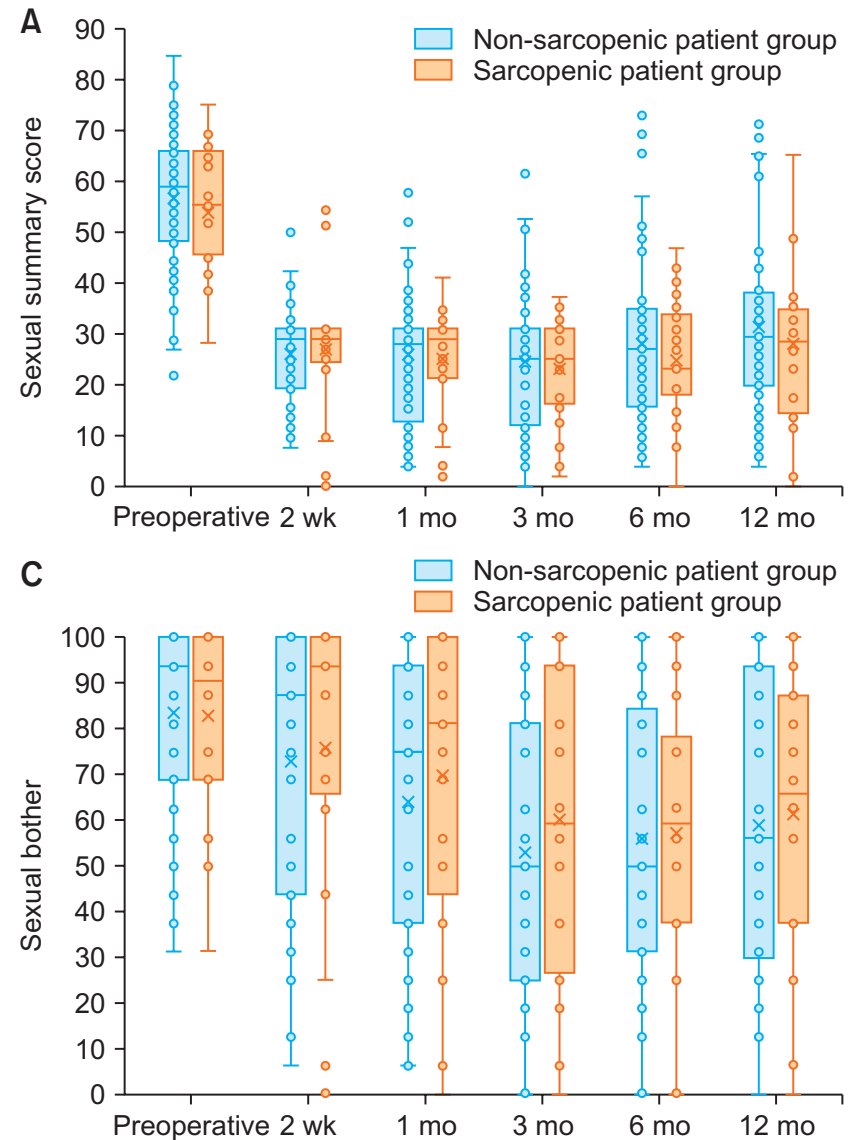

5 as predictors of ED after surgery $(\mathrm{p}=0.035, \mathrm{p}=0.004$, and $\mathrm{p}=0.006$, respectively). Multivariate analysis also revealed that SMI and preoperative IIEF-5 were independent predictors of postoperative ED (odds ratio $[\mathrm{OR}]=3.91, \mathrm{p}=0.1842, \mathrm{OR}=1.13, \mathrm{p}=0.0015$, and $\mathrm{OR}=1.15$, $\mathrm{p}=0.0016$, respectively).

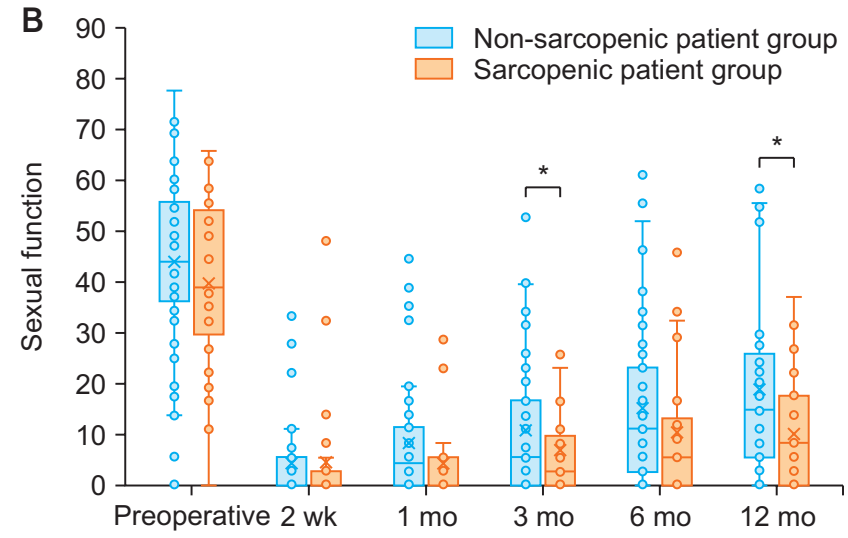

Fig. 2. Change in health-related quality of life after robot-assisted radical prostatectomy. Longitudinal change of Expanded Prostate Cancer Index Composite score. (A) The graph shows sexual summary score. (B) The graph shows sexual function. (C) The graph shows sexual bother. ${ }^{*}$ Statistical significance $(p<0.05)$.

\section{Factors associated with erectile dysfunction at 12 months}

To assess the associations between ED at 12 months and other various factors, univariate and multivariate analyses were performed. Univariate analyses identified the following two factors as significantly associated with a low IIEF-5 at 12 months after RARP: pre- 


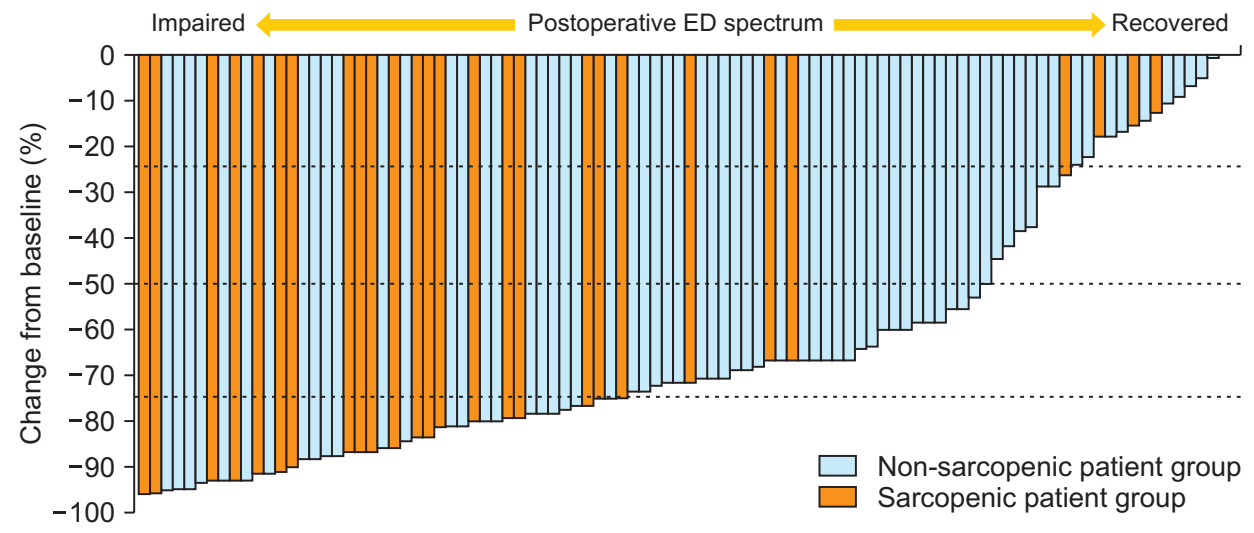

Fig. 3. Change in 5-item version of the International Index of Erectile Function (IIEF-5) after robot-assisted radical prostatectomy (RARP). This water plot shows that change in IIEF-5 from baseline until 12 months after RARP. Total 19 sarcopenic patients $(70.3 \%)$ worse postoperative IIEF- 5 over $75 \%$ while sarcopenic patients were 23 patients (33.8\%).

Table 2. Associations with erectile dysfunction (ED) at 12 months after robot-assisted radical prostatectomy

\begin{tabular}{|c|c|c|c|c|c|c|}
\hline \multirow{2}{*}{ Variable } & \multirow{2}{*}{ Non-ED $(n=51)$} & \multirow{2}{*}{$\operatorname{ED}(n=44)$} & \multirow{2}{*}{$p$-value } & \multicolumn{3}{|c|}{ Multivariate analysis } \\
\hline & & & & OR & $95 \% \mathrm{Cl}$ & p-value \\
\hline Age (y) & $64.5(57-69)$ & $65(61-68)$ & 0.423 & & & \\
\hline BMI $\left(\mathrm{kg} / \mathrm{m}^{2}\right)$ & $24.6(22.9-26.0)$ & $23.9(22.0-25.6)$ & 0.275 & & & \\
\hline $\mathrm{PV}(\mathrm{mL})$ & $24.0(18.7-32.0)$ & $25.0(19.0-37.0)$ & 0.704 & & & \\
\hline PSA (ng/mL) & $7.0(5.1-9.4)$ & $7.5(5.5-9.2)$ & 0.560 & & & \\
\hline Bilateral-NS & 13 & 6 & 0.198 & & & \\
\hline Posterior approach & 7 & 2 & 0.170 & & & \\
\hline Additional treatment & 1 & 6 & 0.035 & 3.91 & $0.41-36.9$ & 0.1842 \\
\hline $\operatorname{VAT}\left(\mathrm{cm}^{2}\right)$ & $128(80-187)$ & $134(83-172)$ & 0.946 & & & \\
\hline $\mathrm{SAT}\left(\mathrm{cm}^{2}\right)$ & $97(80-127)$ & $91(71-127)$ & 0.560 & & & \\
\hline VAT/SAT ratio & $1.1(1.0-1.7)$ & $1.3(0.9-1.7)$ & 0.762 & & & \\
\hline $\mathrm{SM}\left(\mathrm{cm}^{2}\right)$ & $144(133-154)$ & $135(124-150)$ & 0.028 & & & \\
\hline $\mathrm{SMI}\left(\mathrm{cm}^{2} / \mathrm{m}^{2}\right)$ & $52.4(48.2-56.4)$ & $48.0(44.2-52.8)$ & 0.004 & 1.13 & $1.05-1.23$ & 0.0015 \\
\hline Preoperative IIEF-5 & $17(15-22)$ & $15(10-19)$ & 0.006 & 1.15 & $1.05-1.26$ & 0.0016 \\
\hline Use PDE5 inhibitor & 17 & 10 & 0.362 & & & \\
\hline Regularly prescription of PDE5 inhibitor & 9 & 4 & 0.251 & & & \\
\hline
\end{tabular}

Values are presented as median (range) or number (\%).

OR: odds ratio, Cl: confidence interval, BMI: body mass index, PV: prostate volume, PSA: prostate-specific antigen, NS: nerve-sparing, VAT: visceral adipose tissue, SAT: subcutaneous adipose tissue, SM: skeletal muscle, SMI: skeletal muscle index, IIEF-5: 5-item version of the International Index of Erectile Function, PDE5: phosphodiesterase-5.

operative IIEF-5 ( $>16$ vs. $\leq 16$; OR=2.55, 95\% confidence interval [C]: 1.11-5.87, $\mathrm{p}=0.028$ ) and sarcopenia (yes vs. no; OR=3.23, 95\% CI: $1.27-8.25$, $\mathrm{p}=0.014$ ) (Table 3). These two factors were included in a multivariate analysis, which identified preoperative IEF-5 and sarcopenia as significant independent predictors of ED after RARP $(\mathrm{OR}=2.83,95 \%$ CI: 1.18-6.82, $\mathrm{p}=0.0202$ and $\mathrm{OR}=3.559$, 95\% CI: 1.35-9.56, $\mathrm{p}=0.0105$, respectively).

\section{DISCUSSION}

This study identified the presence of preoperative sarcopenia as a novel factor affecting ED recovery and which lowers sexual QoL after RARP. In previous studies, patients' age and preoperative IIEF-5 have been reported as factors associated with ED after RP [6,7]. Our study also established that preoperative IIEF-5 was strongly associated with postoperative SF; however, the age of patients had no relationship with postoperative ED. Although a report has revealed an association between sarcopenia and ED in patients with type 2 diabetes mellitus (DM), no reports link sarcopenia and ED in patients who undergo RARP [17]. It is the authors' understanding, that the study described herein is the first report to demonstrate a relationship between sarcopenia and sexual QoL after RARP. 
Table 3. Associations with sexual dysfunction at 12 months after robot-assisted radical prostatectomy

\begin{tabular}{|c|c|c|c|c|c|c|}
\hline & \multicolumn{6}{|c|}{ ED at 12 months after robot-assisted radical prostatectomy } \\
\hline & \multicolumn{3}{|c|}{ Univariate analysis } & \multicolumn{3}{|c|}{ Multivariate analysis } \\
\hline & OR & $95 \% \mathrm{Cl}$ & p-value & OR & $95 \% \mathrm{Cl}$ & p-value \\
\hline Age & 0.96 & $0.90-1.03$ & 0.239 & & & \\
\hline $\mathrm{BMI} \geq 25 \mathrm{~kg} / \mathrm{cm}^{2}$ & 0.75 & $0.33-1.72$ & 0.502 & & & \\
\hline HT (yes) & 1.01 & $0.42-2.44$ & 0.989 & & & \\
\hline DM (yes) & 0.92 & $0.23-3.66$ & 0.906 & & & \\
\hline PV & 1.00 & $0.96-1.03$ & 0.924 & & & \\
\hline PSA & 1.04 & $0.96-1.12$ & 0.334 & & & \\
\hline Bilateral-NS (no) & 2.17 & $0.75-6.30$ & 0.155 & & & \\
\hline Additional treatment (yes) & 7.54 & $0.87-65.3$ & 0.067 & & & \\
\hline Preoperative IIEF-5 $\leq 16$ & 2.55 & $1.11-5.87$ & 0.028 & 2.83 & $1.18-6.82$ & 0.0202 \\
\hline VAT/SAT ratio $\geq 1.2$ & 1.44 & $0.64-3.25$ & 0.375 & & & \\
\hline Sarcopenia (yes) & 3.23 & $1.27-8.25$ & 0.014 & 3.56 & $1.35-9.56$ & 0.0105 \\
\hline Use PDE5 inhibitor (yes) & 0.58 & $0.24-1.47$ & 0.251 & & & \\
\hline
\end{tabular}

ED: erectile dysfunction, OR: odds ratio, Cl: confidence interval, BMI: body mass index, HT: hypertension, DM: diabetes mellitus, PV: prostate volume, PSA: prostate-specific antigen, NS: nerve-sparing, IIEF-5: 5-item version of the International Index of Erectile Function, VAT: visceral adipose tissue, SAT: subcutaneous adipose tissue, PDE5: phosphodiesterase-5.

Erectile function is dependent on a complex interaction of vascular and neural processes, particularly with respect to increasing blood flow into the corpora cavernosa [14]. Therefore, ED can result from any process that impairs the vascular pathways that contribute to erection [14]. A recent study showed that men with peripheral arterial disease demonstrated a high prevalence of sarcopenia and decreased functional mobility [18]. Sarcopenia can affect postoperative ED. Our findings suggest that an evaluation of sarcopenia before treatment is useful for predicting postoperative ED. ED is defined as the consistent or recurrent inability to attain and/or maintain penile erection sufficient for sexual satisfaction according to the Fourth International Consultation on Sexual Medicine [19]. As we have described previously, aging is an independent risk factor for the development of ED, and many men assume that sexual impairment is an inevitable consequence of aging [14,20]. Risk factors for developing ED include tobacco use, obesity, sedentary lifestyle, and chronic alcohol use. These risk factors would cause hormonal changes that result in low testosterone and impaired endothelial function, which can contribute to the development of ED [14,20]. In contrast, a previous study reported no relationship between metabolic syndrome and ED in healthy men [20]. However, patients who have previously been diagnosed with DM, HT, dyslipidemia, or depression have a higher than normal risk of developing ED [14,20]. Among men diagnosed with ED, approximately $40 \%$ have HT, $42 \%$ have hyperlipidemia, and $20 \%$ have DM [21,22]. ED after RP is recognized as one of the major postoperative complications that considerably impacts the QoL of patients. According to previous reports, initiating a penile rehabilitation program as promptly as possible postsurgery is a better option than inaction, in order to limit or prevent local hypoxygenation and fibrosis [5]. The effectiveness of PDE5 inhibitor therapy on postoperative ED has made it the standard of care treatment for penile rehabilitation $[5,23]$. In this study, we prescribed PDE5 inhibitors, including sildenafil, vardenafil, and tadalafil, in accordance with each patient's preference. No correlation was observed between postoperative ED recovery and PDE5 inhibitor use. Moreover, aging, obesity, HT, and DM were not identified as independent risk factors for postoperative ED. Thus, the cause of postoperative ED was considered to be affected by other factors. Our study revealed that sarcopenia is a novel predictor of postoperative ED.

The incidence of sarcopenia is $20 \%$ in healthy people less than 70 years of age, and more than $50 \%$ after 80 years of age [24]. In our study, $28.4 \%$ of patients were defined as sarcopenic and this result is highly similar to previous reports. As people live longer, the number of patients with sarcopenia will increase [9]. Aging tends to increase the likelihood of SM loss, fat accu- 
mulation, HT, and DM. Previous studies report that obesity and arteriosclerosis, as well as HT and DM, can contribute to the root cause of $\mathrm{ED}[20,25]$. However, the association between sarcopenia and ED after prostatectomy was unknown. In this study, patients with sarcopenia had a higher rate of HT and a greater VAT area in L3 than in the patients without sarcopenia. These factors were not associated with postoperative ED in our multivariate analysis. Instead, we identified preoperative IIEF-5 and sarcopenia as risk factors predictive for ED after prostatectomy. Recently, we investigated the correlation between sarcopenia and urinary incontinence (UIN), and uncovered new evidence showing that the presence of sarcopenia is one factor predictive of UIN after RARP [26]. Thus, the potential for preoperative sarcopenia to be associated with postoperative QoL after RARP is substantial.

It is clinically beneficial to evaluate sarcopenia. At our institution, almost all patients who are diagnosed with PCa undergo CT to screen for potential metastasis. Sarcopenia was diagnosed using CT to measure SM area. Our patients incurred no associated costs, no radiation, and the time burden was negligible. The strength of this diagnostic method is based on the use of automated software that greatly minimizes measurement bias and generates reproducible measurements of SM area, even among multiple observers [10]. Given the long-term survival expectancy of patients with localized $\mathrm{PCa}$, the treatment for PCa should be chosen based on both the oncologic outcome and postoperative QoL. Brachytherapy was shown to be superior to bilateral NS RARP for maintaining, sexual QoL, and treating UIN in one study [27]. Young men in particular, who are eligible for active surveillance, are advised to choose the optimal treatment that takes into consideration postoperative QoL [6]. We identified sarcopenia as a predictor of lower sexual QoL after RARP. Use of a combination of factors, including sarcopenia status, will likely increase the efficacy of predicting ED. These suggest that knowing that before surgery would only help inform treatment decisions - i.e., avoid surgery for low risk disease or just help informed decision making about the decision to forego NS and help patients mentally prepare for post op ED.

This study has some limitations. First, it was a retrospective study performed at a single institution, and the number of patients assessed was relatively low. It is possible that small sample size studies can result in lack of statistical significance due to the relationship between sarcopenia and erectile function. To more conclusively investigate the prognostic role of sarcopenia in QoL, studies involving larger cohorts are necessary. Second, it was well known that testosterone and PCa have a positive association [28,29]. And testosterone would correlate with erectile function [30]. However, we have no data of serum testosterone level. Third, we evaluated sarcopenia using CT scan. To define sarcopenia more clearly, other evaluating methods were needed: hand grip test or walking speed measurement.

Finally, we prescribed PDE5 inhibitors in accordance with the preference of each patient. Some patient continued to use PDE5 inhibitors, whereas others stopped using them because of ineffectiveness. Despite these limitations, this study demonstrated for the first time that preoperative sarcopenia, as measured by $\mathrm{CT}$, is a novel predictor of ED after RARP.

\section{CONCLUSIONS}

After RARP, patients with sarcopenia had a lower sexual QoL compared with patients without sarcopenia at 12 months. Sarcopenia was identified as an independent prognostic factor for postoperative ED recovery. The results of this study contribute to informing urologist regarding treatment options for patients with $\mathrm{PCa}$, although further research is warranted to further clarify the effect of sarcopenia on postoperative SF.

\section{ACKNOWLEDGEMENTS}

The authors thank the clinical laboratory technicians of Okayama University Hospital for their technical support.

\section{Conflict of Interest}

The authors have nothing to disclose.

\section{Author Contributions}

Conceptualization: YM, AGHR, TS. Data curation: YM, YM. Formal analysis: YM, RS. Supervision: KW, MA, MW, TW, YN. Writing - original draft: YM. Writing - review \& editing draft: all authors. 


\section{MEN's HEALTH}

\section{Supplementary Materials}

Supplementary materials can be found via https://doi. org/10.5534/wjmh.200036.

\section{Data Sharing Statement}

The data required to reproduce these findings cannot be shared at this time due to legal and ethical reasons.

\section{REFERENCES}

1. Joglekar S, Nau PN, Mezhir JJ. The impact of sarcopenia on survival and complications in surgical oncology: a review of the current literature. J Surg Oncol 2015;112:503-9.

2. Simonsen C, de Heer P, Bjerre ED, Suetta C, Hojman P, Pedersen BK, et al. Sarcopenia and postoperative complication risk in gastrointestinal surgical oncology: a meta-analysis. Ann Surg 2018;268:58-69.

3. Nishigori T, Okabe H, Tanaka E, Tsunoda S, Hisamori S, Sakai Y. Sarcopenia as a predictor of pulmonary complications after esophagectomy for thoracic esophageal cancer. J Surg Oncol 2016;113:678-84.

4. Mayr R, Fritsche HM, Zeman F, Reiffen M, Siebertz L, Niessen C, et al. Sarcopenia predicts 90 -day mortality and postoperative complications after radical cystectomy for bladder cancer. World J Urol 2018;36:1201-7.

5. Salonia A, Burnett AL, Graefen M, Hatzimouratidis K, Montorsi F, Mulhall JP, et al. Prevention and management of postprostatectomy sexual dysfunctions. Part 1: choosing the right patient at the right time for the right surgery. Eur Urol 2012; 62:261-72.

6. Lee JK, Sjoberg DD, Miller MI, Vickers AJ, Mulhall JP, Ehdaie B. Improved recovery of erectile function in younger men after radical prostatectomy: Does it justify immediate surgery in low-risk patients? Eur Urol 2018;73:33-7.

7. Alemozaffar M, Regan MM, Cooperberg MR, Wei JT, Michalski JM, Sandler HM, et al. Prediction of erectile function following treatment for prostate cancer. JAMA 2011;306:120514.

8. Garcia FJ, Violette PD, Brock GB, Pautler SE. Predictive factors for return of erectile function in robotic radical prostatectomy: case series from a single centre. Int J Impot Res 2015;27:29-32.

9. Larsson L, Degens H, Li M, Salviati L, Lee YI, Thompson W, et al. Sarcopenia: aging-related loss of muscle mass and function. Physiol Rev 2019;99:427-511.

10. Mitsui Y, Sadahira T, Araki M, Maruyama Y, Wada K, Tani-
Yosuke Mitsui, et al: Impact of Sarcopenia on Erectile Function

moto R, et al. Loss of psoas major muscle volume during systemic chemotherapy is related to worse prognosis in testicular cancer. Jpn J Clin Oncol 2019;49:183-9.

11. Martin L, Birdsell L, Macdonald N, Reiman T, Clandinin MT, McCargar LJ, et al. Cancer cachexia in the age of obesity: Skeletal muscle depletion is a powerful prognostic factor, independent of body mass index. J Clin Oncol 2013;31:1539-47.

12. Porpiglia F, Fiori C, Bertolo R, Manfredi M, Mele F, Checcucci E, et al. Five-year outcomes for a prospective randomised controlled trial comparing laparoscopic and robot-assisted radical prostatectomy. Eur Urol Focus 2018;4:80-6.

13. Skolarus TA, Dunn RL, Sanda MG, Chang P, Greenfield TK, Litwin MS, et al.; PROSTQA Consortium. Minimally important difference for the Expanded Prostate Cancer Index Composite Short Form. Urology 2015;85:101-5.

14. Irwin GM. Erectile dysfunction. Prim Care 2019;46:249-55.

15. Savera AT, Kaul S, Badani K, Stark AT, Shah NL, Menon M. Robotic radical prostatectomy with the "Veil of Aphrodite" technique: histologic evidence of enhanced nerve sparing. Eur Urol 2006;49:1065-73; discussion 1073-4.

16. Sadahira T, Mitsui Y, Araki M, Maruyama Y, Wada K, Edamura $\mathrm{K}$, et al. Pelvic magnetic resonance imaging parameters predict urinary incontinence after robot-assisted radical prostatectomy. Low Urin Tract Symptoms 2019;11:122-6.

17. Uçak S, Sivritepe R, Kara O, Sevim E, Ortaboz D, Küçük EV, et al. Association between sarcopenia and erectile dysfunction in males with type II diabetes mellitus. Aging Male 2019;22: 20-7.

18. Addison O, Prior SJ, Kundi R, Serra MC, Katzel LI, Gardner AW, et al. Sarcopenia in peripheral arterial disease: prevalence and effect on functional status. Arch Phys Med Rehabil 2018; 99:623-8.

19. McCabe MP, Sharlip ID, Atalla E, Balon R, Fisher AD, Laumann E, et al. Definitions of sexual dysfunctions in women and men: a consensus statement from the fourth International Consultation on Sexual Medicine 2015. J Sex Med 2016;13: 135-43.

20. Shamloul R, Ghanem H. Erectile dysfunction. Lancet 2013; 381:153-65.

21. Selvin E, Burnett AL, Platz EA. Prevalence and risk factors for erectile dysfunction in the US. Am J Med 2007;120:151-7.

22. Seftel AD, Sun P, Swindle R. The prevalence of hypertension, hyperlipidemia, diabetes mellitus and depression in men with erectile dysfunction. J Urol 2004;171(6 Pt 1):2341-5.

23. Liu C, Lopez DS, Chen M, Wang R. Penile rehabilitation therapy following radical prostatectomy: a meta-analysis. J Sex Med 2017;14:1496-503.

24. Shafiee G, Keshtkar A, Soltani A, Ahadi Z, Larijani B, Heshmat 
R. Prevalence of sarcopenia in the world: a systematic review and meta- analysis of general population studies. J Diabetes Metab Disord 2017;16:21.

25. Gandaglia G, Briganti A, Jackson G, Kloner RA, Montorsi F, Montorsi P, et al. A systematic review of the association between erectile dysfunction and cardiovascular disease. Eur Urol 2014;65:968-78.

26. Mitsui Y, Sadahira T, Watanabe T, Araki M, Maruyama Y, Sato R, et al. Correlation between lumbar skeletal muscle size and urinary incontinence after radical prostatectomy. Low Urin Tract Symptoms 2020. doi: 10.1111/luts.12312 [Epub].

27. Blanchard P, Davis JW, Frank SJ, Kim J, Pettaway CA, Pugh
TJ, et al. Quality of life after brachytherapy or bilateral nervesparing robot-assisted radical prostatectomy for prostate cancer: a prospective cohort. BJU Int 2018;121:540-8.

28. Shin MJ, Jeon YK, Kim IJ. Testosterone and sarcopenia. World J Mens Health 2018;36:192-8.

29. Bell MA, Campbell JD, Joice G, Sopko NA, Burnett AL. Shifting the paradigm of testosterone replacement therapy in prostate cancer. World J Mens Health 2018;36:103-9.

30. Nam YS, Lee G, Yun JM, Cho B. Testosterone replacement, muscle strength, and physical function. World J Mens Health 2018;36:110-22. 